\title{
Electrocardiographic Criteria for Left Ventricular Hypertrophy in Children
}

\author{
Peter R. Rijnbeek - Gerard van Herpen - Livia Kapusta - A. Derk Jan ten Harkel · \\ Maarten Witsenburg $\cdot$ Jan A. Kors
}

Received: 6 November 2007/Accepted: 24 March 2008/Published online: 25 April 2008

(C) The Author(s) 2008

\begin{abstract}
Previous studies to determine the sensitivity of the electrocardiogram (ECG) for left ventricular hypertrophy $(\mathrm{LVH})$ in children had their imperfections: they were not done on an unselected hospital population, several criteria used in adults were not applied to children, and obsolete limits of normal for the ECG parameters were used. Furthermore, left ventricular mass (LVM) was taken as the reference standard for $\mathrm{LVH}$, with no regard for other clinical evidence. The study population consisted of 832 children from whom a 12-lead ECG and an M-mode echocardiogram were taken on the same day. The validity of the ECG criteria was judged on the basis of an abnormal LVM index, either alone or in combination with other clinical evidence. The ECG criteria were based on recently established age-dependent normal limits. At $95 \%$ specificity, the ECG criteria have low sensitivities $(<25 \%)$ when an elevated LVM index is taken as the reference for LVH. When clinical evidence is also taken into account, the sensitivity improved considerably $(<43 \%)$. Sensitivities could be further improved when ECG parameters were combined. The sensitivity of the pediatric ECG in detecting $\mathrm{LVH}$ is low but depends strongly on the definition of the reference used for validation.
\end{abstract}

P. R. Rijnbeek $(\bowtie)$ · G. van Herpen · J. A. Kors Department of Medical Informatics, Erasmus University Medical Center, P.O. Box 2040, Rotterdam 3000 CA, The Netherlands

e-mail: p.rijnbeek@erasmusmc.nl

\section{Kapusta}

Children's Heart Center, Radboud University Medical Center, Nijmegen, The Netherlands

A. D. J. ten Harkel · M. Witsenburg Department of Pediatric Cardiology, Erasmus University Medical Center, Rotterdam, The Netherlands
Keywords Electrocardiography $\cdot$ Pediatrics . Left ventricular hypertrophy · Echocardiography

\section{Introduction}

Left ventricular hypertrophy $(\mathrm{LVH})$ results from adaptation of the heart to increased haemodynamic burden. Therefore, early detection of LVH is important, especially in children. Although the 12-lead electrocardiogram (ECG) is still valued as an initial diagnostic test for $\mathrm{LVH}$, its sensitivity in this respect leaves to be desired. In a recent large study on HIV-infected children, Rivenes et al. [19] found sensitivities of $<20 \%$ at specificity levels of $88 \%$ to $92 \%$. On the other hand, in a number of smaller studies on patients with a specific cardiac disease, the performance of the pediatric ECG was found to be higher [7, 10, 12, 16, 21]. For example, in a group of 19 aortic stenosis patients and 21 normals, Fogel et al. [7] found a sensitivity of $67 \%$ at $95 \%$ specificity. None of these studies was done on an unselected pediatric hospital population with mixed cardiac abnormalities. For the present investigation we have collected such a population and have sought to improve the sensitivity of the pediatric ECG in detecting LVH by means that issue from the following considerations:

First, in pediatric electrocardiology, only a limited number of criteria have been used for assessing LVH. Several criteria that were shown to improve the detection of LVH in adults have not been tested in children. Pediatric electrocardiographers have focused on the QRS amplitude, although the time-voltage area of the QRS complex or its approximation by the product of maximum QRS voltage and QRS duration was proposed in adults as a useful criterion to improve LVH diagnosis [14]. Likewise, in adults combinations of criteria have been shown to improve 
performance [20, 22, 23], which approach has not yet been attempted for children.

Second, older validity studies have used obsolete limits of normal for the pediatric ECG parameters. In a previous study [17], we established new age-dependent normal limits that differ considerably from the older figures [4, 6]. Hitherto, normal limits have not been revised for $\mathrm{LVH}$ detection.

Finally, the reference standard for LVH on the ECG has usually been the left ventricular mass (LVM) as estimated from echocardiographic measurements. This reference has been criticized for being vulnerable to measurement error and for its oversimplification of the geometry of the left ventricle [1, 3]. Alternatively, a combination of increased LVM and clinical evidence of volume or pressure overload of the left ventricle may be a better reference standard for the validity of ECG criteria.

\section{Materials and Methods}

\section{Study Population}

We collected data on all 904 children from whom a 12-lead electrocardiogram and an echocardiogram were taken on the same day at the Sophia Children's Hospital in Rotterdam in the period 2003 to 2005 . We excluded 5 children who had received heart transplantation and 67 children with complete transposition of the great arteries, leaving a study population of 832 children. The age and sex distribution of the population are reported In Table 1.

Clinical data were studied by two pediatric cardiologists (M. W. and A. D. J. H.) who were independently presented with the medical record of each patient. Each cardiologist had to score the likelihood of volume or pressure overload of the right and/or left ventricle on a 3-point scale ( 0 = absent, $1=$ possible, $2=$ probable $)$. LVH was considered present in patients with, e.g., aortic stenosis or regurgitation, and $\mathrm{RVH}$ in patients with tetralogy of Fallot

Table 1 Age and sex distribution of the study population

\begin{tabular}{lccc}
\hline Age & Males & Females & Total \\
\hline $0-2$ mo & 14 & 18 & 32 \\
$3-5$ mo & 15 & 8 & 23 \\
$6-11 \mathrm{mo}$ & 18 & 13 & 31 \\
$1-2$ yr & 50 & 45 & 95 \\
$3-4 \mathrm{yr}$ & 66 & 47 & 113 \\
$5-7 \mathrm{yr}$ & 77 & 65 & 142 \\
$8-11 \mathrm{yr}$ & 106 & 82 & 188 \\
$12-15 \mathrm{yr}$ & 109 & 99 & 208 \\
Total & 455 & 377 & 832 \\
\hline
\end{tabular}

or pulmonary hypertension. Disagreements, defined as a $>1$-point scoring difference, were settled by consensus.

\section{Electrocardiography}

Twelve-lead ECGs were recorded by means of a PC-based acquisition system (Welch Allyn Cardio Control, Delft, The Netherlands) at a sampling rate of $1200 \mathrm{~Hz}$. Following common practice at the Department of Pediatric Cardiology in Rotterdam, V3 was moved to the V3R position, and V5 was moved to the V7 position. All ECGs were processed by the pediatric ECG computer program PEDMEANS [18]. To reduce noise, PEDMEANS computes a representative averaged beat for each of the 12 leads, from which ECG measurements are derived. Wave onsets and offsets as found by PEDMEANS were visually checked.

A total of 15 ECG parameters for diagnosing $\mathrm{LVH}$ in children were evaluated (Table 2). In addition to the standard pediatric parameters, we included parameters based on V3R and V7 and defined an additional version of the Sokolow-Lyon criterion by using V3R and V7 instead of V1 and V6. Furthermore, the sum of the R- and S-wave amplitudes in all leads (12-lead sum) and the QRS voltage-duration product and voltage-time integral versions of the Sokolow-Lyon and 12-lead sum criteria were added. The sensitivities of the voltage-duration product version and the voltage-time integral version were compared with their amplitude versions by means of the McNemar's modification of the chi-square method for paired proportions.

Table 2 Evaluated ECG parameters for left ventricular hypertrophy

\begin{tabular}{|c|c|}
\hline Parameter & Description \\
\hline SV3R & S-wave amplitude in V3R \\
\hline SV1 & S-wave amplitude in V1 \\
\hline RV6 & R-wave amplitude in V6 \\
\hline RV7 & R-wave amplitude in V7 \\
\hline TV6 & Inverted $\mathrm{T}$ wave in $\mathrm{V} 6$ \\
\hline TV7 & Inverted $\mathrm{T}$ wave in $\mathrm{V} 7$ \\
\hline $\mathrm{SV} 1+\mathrm{RV} 6$ & Sokolow-Lyon voltage \\
\hline$(\mathrm{SV} 1+\mathrm{RV} 6) \times \mathrm{QRS}_{\mathrm{d}}$ & Sokolow-Lyon voltage-duration product \\
\hline$(\mathrm{SV} 1+\mathrm{RV} 6)$ area & Sokolow-Lyon voltage-time integral \\
\hline SV3R + RV7 & Additional Sokolow-Lyon voltage \\
\hline$(\mathrm{SV} 3 \mathrm{R}+\mathrm{RV7}) \times \mathrm{QRS}_{\mathrm{d}}$ & $\begin{array}{l}\text { Additional Sokolow-Lyon voltage- } \\
\text { duration product }\end{array}$ \\
\hline$(\mathrm{SV} 3 \mathrm{R}+\mathrm{RV7})$ area & $\begin{array}{l}\text { Additional Sokolow-Lyon voltage-time } \\
\text { integral }\end{array}$ \\
\hline 12-lead sum & Sum of top-top deflections of all leads \\
\hline 12-lead sum x $\mathrm{QRS}_{\mathrm{d}}$ & 12-lead sum voltage-duration product \\
\hline 12-lead sum area & 12-lead sum voltage-time integral \\
\hline
\end{tabular}


The polarity of the $\mathrm{T}$ wave in V6 or V7 was taken as a binary value. For all other parameters, reference values were derived from a normal population of 1912 children aged 11 days to 16 years [17]. The 98th percentile of the parameter distribution was taken as the upper limit of normal (ULN). We estimated age-dependent percentile curves using a two-stage parametric approach described before [17]. As an illustration, the age-dependent normal curve for the 12-lead sum is presented in Fig. 1. For each LVH parameter, the difference between the parameter value and its corresponding normal limit was taken for further processing.

\section{Echocardiography}

The echocardiograms were recorded with a Philips Sonos 5500 (Philips, Best, The Netherlands). The following Mmode measurements were obtained according to the American Society of Echocardiographers' convention [9]: interventricular septum thickness at end diastole (IVSd), posterior wall thickness at end diastole (PWLVd), and left ventricular internal dimension at end diastole (LVIDd).

Left ventricular mass (LVM) was calculated with the anatomically validated formula of Devereux [5]:

$$
\begin{aligned}
& \mathrm{LVM}=0.8 \times[1.04 \\
& \quad \times\left(\mathrm{IVSd}+\mathrm{PWLVd}+\mathrm{LVIDd}^{3}-\mathrm{LVIDd}^{3}\right]+0.6[\mathrm{~g}]
\end{aligned}
$$

The LVM index (LVMI) adjusts for body size and is taken as LVM ( $\mathrm{g}$ ) divided by body weight $(\mathrm{kg})$. As shown in Fig. 2, we estimated a partition value of 3 for LVMI, based on a set of 587 normal echocardiograms of 361 boys and 226 girls aged birth to 18 years previously described by Overbeek et al. [15]. We calculated the LVM using the

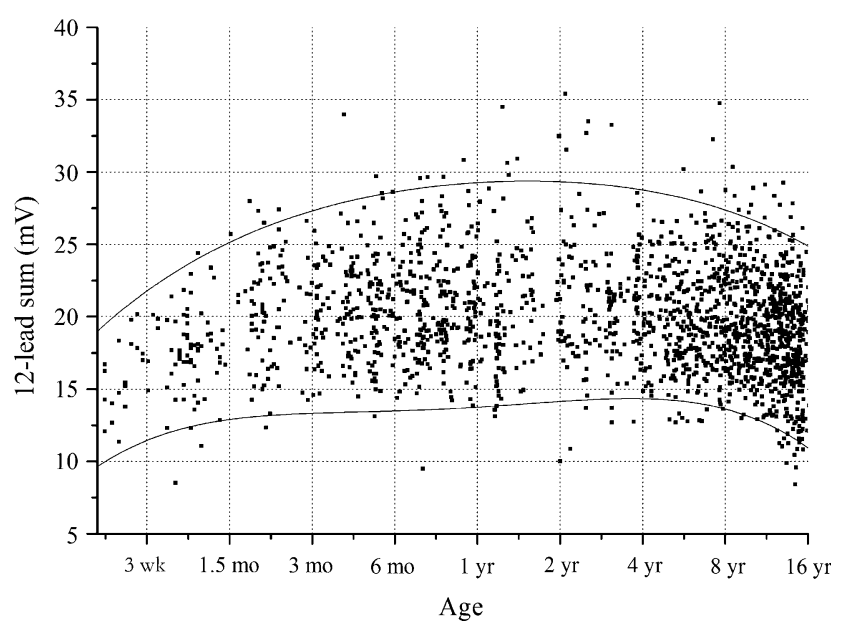

Fig. 1 Scatter diagram for the 12-lead sum versus age. The solid lines indicate the 2 nd and 98 th percentile curves

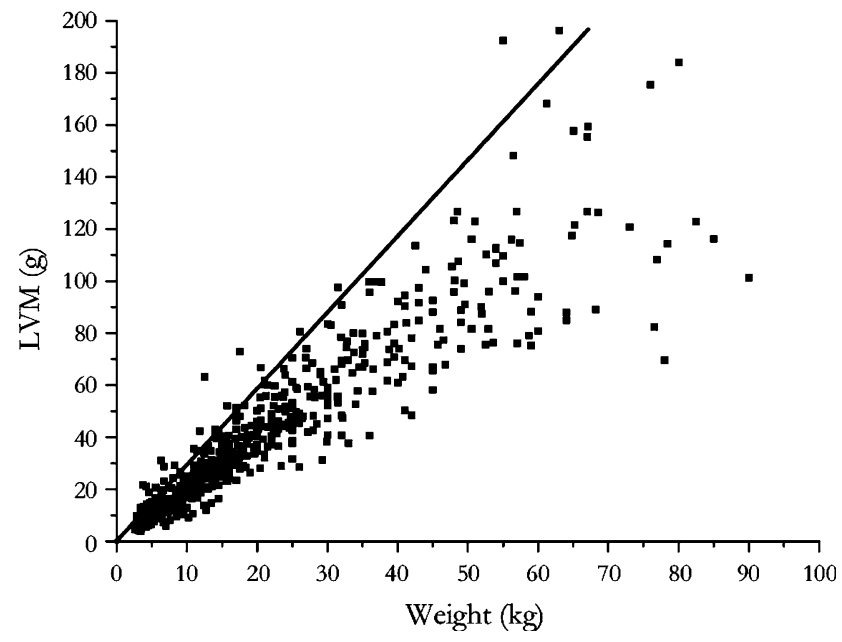

Fig. 2 Left ventricular mass (LVM) calculated on 587 normal echocardiograms plotted against body weight. The solid line represents the partition value of the LVM index, $\mathrm{LVM}_{\mathrm{I}}=\mathrm{LVM} /$ weight $=3$

formula of Devereux from the measurements provided to us by Overbeek.

\section{Reference Standards for LVH}

The ECG criteria for LVH were validated using two different reference standards. As in other validation studies, one standard is based only on the LVMI. LVH was considered present at LVMI $>3$, which is indicated by LVM+. In the second standard the clinical evidence of LVH is also taken into consideration and LVH was defined at two different likelihood levels. LVH was assumed to be present at the first level if LVMI $>3$ and at least one cardiologist scored "possible" (indicated by LVM+, L1), and at the second level if LVMI $>3$ and at least one cardiologist scored "probable" (LVM+, L2). Note that the difference between the two cardiologists can never be higher than 1 scoring point due to the consensus rule. The cases with a LVMI $\leq 3$ were classified as non-LVH.

\section{Combination of ECG Parameters}

Combinations of LVH-ECG parameters were evaluated using our EXPLORE induction algorithm [8]. EXPLORE can search for the decision rule that has the highest sensitivity at a user-specified level of specificity. In this study, we wanted EXPLORE to find the best decision rule consisting of two ECG parameters at a specificity of $95 \%$. EXPLORE takes as its input a set of ECGs, each presented by a set of parameters, and a label indicating whether or not LVH is present. We searched for the best parameter combination for each of the three LVH definitions: LVM+; $\mathrm{LVM}+, \mathrm{L} 1$; and $\mathrm{LVM}+, \mathrm{L} 2$. 


\section{Results}

\section{Performance of Individual LVH-ECG Parameters}

Table 3 reports the diagnostic performance of all ECG parameters for different age groups and for the total study population, taking LVM+ as LVH definition. In the complete set (0-15 years), the additional version of the Sokolow-Lyon parameter (SV3R + RV7) performs best, with $25.3 \%$ sensitivity. Multiplying by QRS duration or taking the voltage-time integral does not improve this criterion. However, there is an increase in sensitivity of $31 \%$ by the area version compared to the amplitude-only version of the 12-lead sum in the complete set $(p=0.045)$.

The effect of age on the results is mixed. For different age groups different parameters perform best, and the improvement of the 12-lead sum is not consistent in all age groups. However, this may be accounted for by the lower number of $\mathrm{LVH}$ cases in the higher age groups. For SV1, SV3R, RV6, RV7, TV6, and (SV1 + RV6) $\times$ QRS duration, an increasing performance is seen with age. In all age groups, SV3R appears to perform better than SV1, and RV7 better than RV6.

Table 4 reports the diagnostic performance of the individual ECG parameters for the different definitions of LVH. The sensitivity of all ECG criteria improves considerably when, apart from LVM+, the clinical evidence of $\mathrm{LVH}$ is also entered in the definition of LVH. The more certain the cardiologists were about the presence of $\mathrm{LVH}$, the better the ECG performs. The additional Sokolow-Lyon

Table 3 Sensitivity (\%) at $95 \%$ specificity for three age groups and for the total population taking left ventricular mass index $>3$ as definition of left ventricular hypertrophy

\begin{tabular}{lllll}
\hline Parameter & $0-5 \mathrm{yr}$ & $6-11 \mathrm{yr}$ & $12-15 \mathrm{yr}$ & $0-15 \mathrm{yr}$ \\
\hline SV3R & 16.5 & 17.1 & 19.4 & 16.7 \\
SV1 & 18.3 & 20.0 & 33.3 & 20.4 \\
RV6 & 12.2 & 15.7 & 16.7 & 16.3 \\
RV7 & 19.1 & 20.0 & 25.0 & 22.2 \\
TV6 & 7.0 & 10.0 & 13.9 & 9.0 \\
TV7 & 17.4 & 14.3 & 25.0 & 15.8 \\
SV1 + RV6 & 20.0 & 22.9 & 19.4 & 22.2 \\
$(\mathrm{SV} 1+\mathrm{RV6}) \times \mathrm{QRS}_{\mathrm{d}}$ & 16.5 & 21.4 & 22.2 & 24.0 \\
$(\mathrm{SV1}+\mathrm{RV6})$ area & 19.1 & 21.4 & 19.4 & 24.4 \\
SV3R + RV7 & 29.6 & 18.6 & 27.8 & 25.3 \\
$(\mathrm{SV} 3 R+\mathrm{RV7}) \times \mathrm{QRS}_{\mathrm{d}}$ & 22.6 & 20.0 & 22.2 & 23.1 \\
$(\mathrm{SV} 3 R+\mathrm{RV7})$ area & 20.0 & 20.0 & 19.4 & 20.8 \\
12-lead sum & 15.7 & 17.1 & 22.2 & 17.2 \\
12-lead sum $\times \mathrm{QRS}_{\mathrm{d}}$ & 19.1 & 17.1 & 22.2 & 18.6 \\
12-lead sum area & 22.6 & 14.3 & 27.8 & 22.6 \\
No. cases (LVH+/LVH-) & $115 / 225$ & $70 / 214$ & $36 / 172$ & $221 / 611$ \\
\hline
\end{tabular}

Table 4 Sensitivity (\%) at 95\% specificity for three left ventricular hypertrophy (LVH) definitions

\begin{tabular}{llll}
\hline Parameter & LVM+ & LVM+, L1 & LVM+, L2 \\
\hline SV3R & 16.7 & 21.1 & 30.0 \\
SV1 & 20.4 & 25.9 & 33.8 \\
RV6 & 16.3 & 19.0 & 26.3 \\
RV7 & 22.2 & 25.9 & 36.3 \\
TV6 & 9.0 & 10.9 & 12.5 \\
TV7 & 15.8 & 20.4 & 26.3 \\
SV1 + RV6 & 22.2 & 25.2 & 35.0 \\
(SV1 + RV6) $\times$ QRS $_{\mathrm{d}}$ & 24.0 & 29.9 & 31.3 \\
$(\mathrm{SV1}+\mathrm{RV6})$ area & 24.4 & 30.6 & 41.3 \\
SV3R + RV7 & 25.3 & 30.6 & 42.5 \\
$(\mathrm{SV} 3 R+\mathrm{RV7}) \times \mathrm{QRS}_{\mathrm{d}}$ & 23.1 & 28.6 & 37.5 \\
$(\mathrm{SV} 3 \mathrm{R}+\mathrm{RV7})$ area & 20.8 & 26.5 & 37.5 \\
12-lead sum & 17.2 & 21.1 & 33.8 \\
12-lead sum $\times$ QRS & 18.6 & 21.8 & 33.8 \\
12-lead sum area & 22.6 & 27.9 & 37.5 \\
No. cases $($ LVH+/LVH-) & $221 / 611$ & $147 / 611$ & $80 / 611$ \\
\hline
\end{tabular}

Note. $\mathrm{LVM}+$, left ventricular mass index $>3$; L1, at least one cardiologist scored possible LVH; L2, at least one cardiologist scored probable $\mathrm{LVH}$

criterion (SV3R + RV7) performs best for all reference standards. Removal of all LVH cases from the LVM+, L2 group which were also marked by at least one cardiologist as probable RVH $(n=15)$ hardly changed the performance of the parameters.

\section{Performance of a Combination of Two LVH-ECG Parameters}

Using the EXPLORE algorithm, we found that for all definitions of $\mathrm{LVH}$ the best rule consisted of (SV3R + RV7) area in conjunction with 12-lead sum area. The threshold values of these parameters were optimized by EXPLORE to ensure $95 \%$ specificity. Table 5 reports that this combination improves the sensitivity by $21 \%-25 \%$ compared to the best single parameter (SV3R + RV7).

Table 5 Sensitivities (\%) for the best single parameter (SV3R + RV7) and the best combination of two parameters ([SV3R + RV7] area with 12-lead sum area) for the three LVH definitions at $95 \%$ specificity

\begin{tabular}{llll}
\hline LVH definition & 1 parameter & 2 parameters & \% increase \\
\hline LVM+ & 25.3 & 31.7 & 25.3 \\
LVM+, L1 & 30.6 & 38.1 & 24.5 \\
LVM+, L2 & 42.5 & 51.3 & 20.7 \\
\hline
\end{tabular}

Note. LVM+, left ventricular mass index $>3$; L1, at least one cardiologist scored possible LVH; L2, at least one cardiologist scored probable LVH 


\section{Discussion}

We performed the first large study on the validity of ECG criteria for detecting LVH in an unselected pediatric hospital population with up-to-date reference standards for both electrocardiographic and echocardiographic parameters. The unreliability of reference standards for both the ECG criteria and the echocardiographic-based criteria may previously have been accountable for the disappointing performance of the ECG. Rivenes et al. [19] demonstrated that the often used standard of normal ECG limits of Davignon et al. [4] needed to be revised. They derived new age-adjusted reference values for their HIV-uninfected group of children $<6$ years old. With these new normal values the sensitivity decreased slightly (from $<20 \%$ to $<17 \%$ ) while the specificity improved (from $88 \%-92 \%$ to 94\%-100\%). Regarding the echocardiogram, a recent study provided echocardiographic dimensions that differed significantly from previous data, probably owing to improved measuring techniques [15].

The sensitivity of the ECG for detecting LVH in children is relatively low when LVM+ is taken as the LVH definition (Table 3). The best single parameter is (SV3R + RV7), with a sensitivity of $25.3 \%$ at $95 \%$ specificity. Notably, SV3R, RV7, and (SV3R + RV7) perform better than SV1, RV6, and (SV1 + RV6), respectively. The best-performing parameter that uses leads from the standard 12-lead ECG is the voltage-time integral version of the Sokolow-Lyon criterion, with a sensitivity of $24.4 \%$. Further, the use of voltage-duration product and voltagetime integral of the 12-lead sum resulted in higher sensitivities than the amplitude-only version in the total population. However, overall the use of voltage-duration products and voltage-time integrals for the diagnosis of LVH remains less effective than in adults.

In most studies on the usefulness of the ECG in detecting LVH, echocardiographic-determined LVM has been taken as the reference standard. However, we believe that the ECG should not only be judged by taking LVM as the reference. LVM determination suffers from large measurement errors in both directions [1, 3]. When underestimated, this will go unnoticed because of the high specificity levels to which the criteria are set. When LVM is overestimated, however, the ECG is penalized for stating normal. We therefore also used combinations of increased LVM and clinical indexes of volume or pressure overload to ascertain the validity of ECG criteria. We found that the ECG performs considerably better when there is more certainty about the presence of LVH on clinical grounds. Fogel et al. [7] showed that the sensitivity was $67 \%$ in a small group of 19 children with aortic stenosis. Unfortunately, we could not collect a large enough group of aortic stenosis patients without other abnormalities to make a better comparison with Fogel's data.

The combination of different ECG LVH parameters is known to improve performance in adults [20, 22, 23]. The combination of highly specific criteria can be a fruitful approach to increase sensitivity without generating an unacceptable number of false-positives. We restricted the search of our EXPLORE algorithm to combinations of only two parameters because more parameters might introduce overspecialization on a data set of our size. A combination of (SV3R + RV7) area and 12-lead sum area was found to improve the sensitivity by $25 \%$ in the LVM+ and LVM+, $\mathrm{L} 1$ groups and by $21 \%$ in the $\mathrm{LVM}+, \mathrm{L} 2$ group, at $95 \%$ specificity. Combination of parameters can thus be useful in the pediatric population also.

Our study has several limitations. First, we validated the ECG criteria for LVH using LVM as measured by M-mode echocardiography. However, there are studies in adults suggesting that cardiac MRI can more accurately and reproducibly measure LVM $[1,11]$. In children this has not yet been confirmed. Second, in our study V3 was moved to the V3R position, and V5 was moved to the V7 position. Therefore, the 12-lead sum included these leads instead of V3 and V5, which makes comparison with other studies using standard lead sets more difficult. Third, we could not determine the effect of concomitant RVH on the performance of the ECG in detecting LVH due to the low number of cases scored as probable RVH by the cardiologists $(n=15)$. Fourth, we could not establish the effect of gender because of the limited size of the study population. In adults gender influences the performance of the ECG criteria for LVH $[1,2,13]$. In our normal pediatric ECG population we did find gender differences in QRS duration for all ages and in R- and S-wave amplitudes for children aged 12 to 16 years [17]. The effect of gender on ECGLVH criteria in the pediatric population awaits investigation on a larger population.

Open Access This article is distributed under the terms of the Creative Commons Attribution Noncommercial License which permits any noncommercial use, distribution, and reproduction in any medium, provided the original author(s) and source are credited.

\section{References}

1. Alfakih K, Walters K, Jones T, Ridgway J, Hall AS, Sivananthan M (2004) New gender-specific partition values for ECG criteria of left ventricular hypertrophy: recalibration against cardiac MRI. Hypertension 44:175-179

2. Casale PN, Devereux RB, Alonso DR, Campo E, Kligfield P (1987) Improved sex-specific criteria of left ventricular hypertrophy for clinical and computer interpretation of electrocardiograms: validation with autopsy findings. Circulation $75: 565-572$ 
3. Crow RS, Hannan P, Grandits G, Liebson P (1996) Is the echocardiogram an appropriate ECG validity standard for the detection and change in left ventricular size? J Electrocardiol 29(Suppl):248-255

4. Davignon A, Rautaharju P, Boisselle E, Soumis F, Megelas M, Choguette A (1979/1980) Normal ECG standards for infants and children. Pediatr Cardiol 1:123-131

5. Devereux RB, Alonso DR, Lutas EM, Gottlieb GJ, Campo E, Sachs I, Reichek N (1986) Echocardiographic assessment of left ventricular hypertrophy: comparison to necropsy findings. Am J Cardiol 57:450-458

6. Dickinson DF (2005) The normal ECG in childhood and adolescence. Heart 91:1626-1630

7. Fogel MA, Lieb DR, Seliem MA (1995) Validity of electrocardiographic criteria for left ventricular hypertrophy in children with pressure- or volume-loaded ventricles: comparison with echocardiographic left ventricular muscle mass. Pediatr Cardiol $16: 261-269$

8. Kors JA, Hoffmann A (1997) Induction of decision rules that fulfil user-specified performance requirements. Pattern Recogn Lett 19:1187-1195

9. Lang RM, Bierig M, Devereux RB, Flachskampf FA, Foster E, Pellikka PA, Picard MH, Roman MJ, Seward J, Shanewise JS, Solomon SD, Spencer KT, Sutton MS, Stewart WJ (2005) Recommendations for chamber quantification: a report from the American Society of Echocardiography's Guidelines and Standards Committee and the Chamber Quantification Writing Group, developed in conjunction with the European Association of Echocardiography, a branch of the European Society of Cardiology. J Am Soc Echocardiogr 18:1440-1463

10. Louie EK, Maron BJ (1986) Hypertrophic cardiomyopathy with extreme increase in left ventricular wall thickness: functional and morphologic features and clinical significance. J Am Coll Cardiol $8: 57-65$

11. Myerson SG, Montgomery HE, World MJ, Pennell DJ (2002) Left ventricular mass: reliability of M-mode and 2-dimensional echocardiographic formulas. Hypertension 40:673-678

12. Oda T, Hamamoto K, Morinaga H (1982) Left ventricular hypertrophy in non-rheumatic myocarditis in children. Jpn Circ J 46:1235-1238

13. Okin PM, Roman MJ, Devereux RB, Kligfield P (1995) Gender differences and the electrocardiogram in left ventricular hypertrophy. Hypertension 25:242-249
14. Okin PM, Roman MJ, Devereux RB, Pickering TG, Borer JS, Kligfield P (1998) Time-voltage QRS area of the 12-lead electrocardiogram: detection of left ventricular hypertrophy. Hypertension 31:937-942

15. Overbeek LI, Kapusta L, Peer PG, de Korte CL, Thijssen JM, Daniels O (2006) New reference values for echocardiographic dimensions of healthy Dutch children. Eur J Echocardiogr 7:113121

16. Panza JA, Maron BJ (1989) Relation of electrocardiographic abnormalities to evolving left ventricular hypertrophy in hypertrophic cardiomyopathy during childhood. Am J Cardiol 63:1258-1265

17. Rijnbeek PR, Witsenburg M, Schrama E, Hess J, Kors JA (2001) New normal limits for the paediatric electrocardiogram. Eur Heart J 22:702-711

18. Rijnbeek PR, Witsenburg M, Szatmari A, Hess J, Kors JA (2001) PEDMEANS: a computer program for the interpretation of pediatric electrocardiograms. J Electrocardiol 34(Suppl):85-91

19. Rivenes SM, Colan SD, Easley KA, Kaplan S, Jenkins KJ, Khan MN, Lai WW, Lipshultz SE, Moodie DS, Starc TJ, Sopko G, Zhang W, Bricker JT (2003) Usefulness of the pediatric electrocardiogram in detecting left ventricular hypertrophy: results from the Prospective Pediatric Pulmonary and Cardiovascular Complications of Vertically Transmitted HIV Infection (P2C2 HIV) multicenter study. Am Heart J 145:716-723

20. Salles G, Leocadio S, Bloch K, Nogueira AR, Muxfeldt E (2005) Combined QT interval and voltage criteria improve left ventricular hypertrophy detection in resistant hypertension. Hypertension 46:1207-1212

21. Sastroasmoro S, Madiyono B, Oesman IN (1991) Sensitivity and specificity of electrocardiographic criteria for left ventricular hypertrophy in children with rheumatic heart disease. Paediatr Indones 31:233-244

22. Schillaci G, Verdecchia P, Borgioni C, Ciucci A, Guerrieri M, Zampi I, Battistelli M, Bartoccini C, Porcellati C (1994) Improved electrocardiographic diagnosis of left ventricular hypertrophy. Am J Cardiol 74:714-719

23. Warner RA, Ariel Y, Gasperina MD, Okin PM (2002) Improved electrocardiographic detection of left ventricular hypertrophy. J Electrocardiol 35(Suppl):111-115 